\title{
Clinical evaluation of children testing positive in screening tests for attention-deficit/hyperactivity disorder: A preliminary report
}

\author{
Maria Skounti PhD* \\ Katerina Mpitzaraki MD** \\ Anastas Philalithis MD ${ }^{\star \star \star}$ \\ Emmanouil Galanakis MD, $\mathrm{PhD}^{\star * \star *}$ \\ * $2^{\text {nd }}$ Special Primary School, Heraklion, \\ Crete \\ ** Venizeleion General Hospital, Heraklion, \\ Crete \\ *** Department of Social Medicine, \\ University of Crete \\ **** Department of Pediatrics, University of \\ Crete \\ GREECE
}

\begin{abstract}
Background and Objectives: Screening tests are of great diagnostic value in attention-deficit/hyperactivity disorder (ADHD), however final diagnosis relies on a clinical examination by an expert. The objective of the present study was to clinically evaluate children who had been screened positive for ADHD through both a parent and a teacher questionnaire.

Methods: Parent interview and child behavior checklist and clinical assessment were used to confirm the preliminary diagnosis in 42 children aged 8 years, who have been screened positive for ADHD out of 1,708 children, in a large, two-setting screening study conducted in Crete, Greece.

Results: The diagnosis of ADHD was confirmed for 31 children (74\%). In the remaining 11 children, ADHD manifestations were attributed to other primary disorders. None of the 42 children was classified as lacking symptoms suggesting ADHD. Among the 31 children with confirmed ADHD, only 2 had been diagnosed prior to the screening test.

Conclusions: Although clinical evaluation is the golden standard for diagnosis of ADHD, two-setting screening questionnaires by parent and teacher are useful tools in identifying children who need further investigation and intervention.
\end{abstract}




\section{Introduction}

Attention-deficit/hyperactivity disorder (ADHD) is among the most common, intensely investigated, and yet diagnostically controversial neurobehavioral conditions of childhood $^{1}$. Recorded prevalence rates worldwide range from $2.4-17.8 \%$, but the majority of epidemiological studies indicates prevalence rates ranging from $4 \%$ to $10 \%^{2,3}$. The core symptoms include inattention, hyperactivity and impulsivity. Three subtypes of ADHD listed in DSM-IV can be diagnosed: the predominantly inattentive type, the predominantly hyperactive-impulsive type and the combined type ${ }^{4}$. ADHD often associated with a variety of cognitive, psychiatric, educational, emotional and social impairments ${ }^{5,6}$. Some of these are a consequence of the disorder while other may arise from other primary disorders that overlap with $\mathrm{ADHD}^{7-9}$.

Several standardized behavior rating scales have been reported to perform well in screening of ADHD. These scales offer a convenience by providing a cross-sectional view of ADHD symptomatology, but definite diagnosis of ADHD is made exclusively on clinical grounds ${ }^{10-12}$. The aim of the present study was to investigate, by making use of a complete clinical assessment, children who had been found positive for ADHD by a large scale initial screening.

\section{Methods}

\section{Subjects}

In a large-scale screening of ADHD among schoolchildren in Crete, Greece, all elementary school children attending the first grade of 55 primary schools were assessed, by using parent and teachers' rating scales. Briefly, among 1,708 children, 84 (6.5\%; 95\% confidence interval, $5.2 \%$ to $7.9 \%$ ) screened positive by both parent and teacher for $\mathrm{ADHD}^{13}$.

Six months after the initial screening, all 84 children ( 58 boys, 26 girls) were called for clinical assessment. Of them, 42 (50\%; 35 males, 7 females) were available. All children were Greek nationals except of two coming from immigrant families from Albania. Only two out of these 42 children had been previously diagnosed as having ADHD. Out of 42 parents who participated themselves in the clinical assessment, $12(28.6 \%)$ had primary education degrees and come from lower socio-economic status, $20(47.6 \%)$ had secondary school education degrees and 10 (23.8\%) had higher education. Among the 42 families who were not available to clinical evaluation, four had moved to other residence, seven informed teachers that they were not eager to participate and the remaining 31 non-responders did not reply at all.

\section{Screening tools-procedures}

The initial screening procedure has been described elsewhere. ${ }^{13}$ Briefly, a 2-stage screening process was employed using Attention Deficit Hyperactivity Disorder test (ADHDT) by Gilliam ${ }^{14}$ and a rating scale of student behavior. ${ }^{15-16}$ Parents completed the ADHD test and teachers the rating scale of student behavior. ADHDT was based on DSM-IV diagnostic criteria; comprised of 36 questions in three subtests related to the three core symptoms of ADHD: hyperactivity, impulsivity and inattention. The test provided important information for the assessment of ADHD, including items investigating the disorder's age of onset, the existence of co-occurent disorders and the settings where ADHD symptomatology was present. Teacher's questionnaire was based on Lerner's behavior rat- 
ing scale, enhance with items touching DSMIV criteria for diagnosis of ADHD and was comprised of 50 items, evaluating auditory comprehension, verbal language, orientation, general behavior, motor behavior, attention, hyperactivity-impulsivity, optical-motor coordination, acoustic processing, memory and academic performance. All children who were screened positive by both parent and teacher questionnaire were called for clinical evaluation. The study was approved by both the Hellenic Ministry of Education and the Institutional Committee of the Faculty of Medicine, University of Crete.

\section{Procedures}

Parents of screening-positive children were informed in written about the nature of the disorder and the potential benefits of their child having a clinical evaluation. Contact telephone numbers, the names and the affiliations of the investigating team, and the venue of the evaluation were also included. The clinic of a child-psychiatrist's was chosen as the place for the clinical evaluation, rather than a hospital milieu, where parents and children might feel less com- fortable. Clinical evaluation was performed by a child psychiatrist (KM) and by a special educator specialized in children with developmental disorders (MS).

\section{Assessment}

For a child to be definitely diagnosed as having ADHD, the criteria of the Diagnostic and Statistical Manual of Psychiatric Disorders (DSM-IV) ${ }^{4}$ were applied. Children were assessed by semi-structured clinical parent interview $^{17}$ (Table I). Clinical evaluation focused on presence of signs and symptoms; child's activities, school, friends, family relations, presence of symptoms suggesting other disorders, such as anxiety, depression, unusual thinking, hallucinations, coordination difficulties, gross and fine motor skills, reading, mathematics, speaking performance according to developmental level (examination performed by special educator), memory and comprehension; left-right recognition and optical-visual coordination. Throughout the interview, notes were kept of the child's behavior. At the end of the evaluation the child was allowed to play, draw or wander around the office.

Table I

Structured clinical interview for the diagnosis of ADHD (Based on Barkley's ADHD clinical parent interview ${ }^{17}$ )

ADHD clinical parent interview comprised of

Developmental history: prenatal and perinatal, developmental milestones.

Medical history: hearing, vision, gross-motor, fine coordination, speech articulation, health problems, infections, accidents, surgeries, bladder and bowel control, eating disorders.

Treatment history: pharmacotherapies and psychotherapies.

School history: academic and social progress, school adaptability.

Social history: interaction and relationships with siblings and friends.

Current behavioral concerns.

Diagnostic criteria of ADHD, oppositional defiant disorder, conduct disorder, anxiety, overanxious disorder, depression, dysthymia, autistic and Asperger disorder.

Family history including information on mental disorders in parents and siblings, marital and employment difficulties, stressful events within the family, family relationships, temperament of parents, family history of ADHD or other disorders. 
Additionally all children were evaluated by Child Behavior Checklist (CBCL) ${ }^{18}$ a standardized parent-report questionnaire comprising of 113 items, for the assessment of multiple domains of children's psychopathology, including affective disorders, anxiety, ADHD, oppositional defiant disorder, somatic and conduct problems. In CBCL the parent indicates whether each item is very true (score of 2 ), somewhat true (score of 1 ), or not true (score of 0). A T-score of 69 or more is indicative for ADHD. The attention subscales, commonly used in the assessment of ADHD, have been shown to demonstrate adequate reliability and criterion-related validity ${ }^{18,19}$.

Differential diagnoses were classified after application of the exclusion criteria (according to criterion E of DSM-IV ${ }^{4}$ for ADHD diagnosis). Exclusion criteria included pervasive developmental disorders (e.g., autistic disorder, Asperger's syndrome), psychotic disorders, mood, anxiety and personality disorders, mental retardation, major neurological disorder (epilepsy, acute infantile hemiplegia, brain injury) and chronic medical conditions. The presence of any of these conditions was determined by the medical, developmental and family history obtained from the parent and by the clinical evaluation of each child.

Other conditions that might be mistaken for ADHD or might co-occur, apart from the disorders referred above ${ }^{12,20}$ were additionally considered before a definite diagnosis was made. Such co-concurrent conditions were identified by a detailed history, by the Child Behavior Checklist scored by parent, from the teacher questionnaire, which was available from the screening procedure and by the clinical evaluation.

\section{Results}

Among the 42 children who were available for clinical evaluation, ADHD diagnosis was confirmed for $31(73.8 \%)$, of whom 25 were boys and 6 were girls. In 22 (18 boys, 4 girls) the final diagnosis was ADHD combined type (ADHD-C), in 5 (all boys) ADHD predominately hyperactive-impulsive type (ADHD-HI) and in 4 (2 boys, 2 girls) ADHD predominately inattentive type (ADHD-I). The diagnosis of ADHD was reconfirmed in the 2 children who had been previously diagnosed as having the disorder.

For 11 children ADHD diagnosis was not confirmed as diagnostic criteria were fulfilled for other primary conditions. The distribution of the clinical diagnosis for all children is depicted in Table II.

Table II

Final clinical diagnosis in 42 ADHD screening-positive children

\begin{tabular}{lrrr} 
Diagnosis & Boys & Girls & Total \\
& $\mathrm{N}$ & $\mathrm{N}$ & $\mathrm{N}(\%)$ \\
\hline ADHD & 25 & 6 & $31(74)$ \\
Developmental, speech, language and learning disorders. & 4 & 1 & $5(12)$ \\
Neurological disorders (epilepsy, acute infantile hemiplegia). & 3 & 0 & $3(7.1)$ \\
Emotional problems (family dysfunction, parental abandonment). & 2 & 0 & $2(4.8)$ \\
Psychiatric developmental disorders (autism). & 1 & 0 & $1(2.4)$ \\
Total & 35 & 7 & $42(100)$ \\
\hline
\end{tabular}




\section{Discussion}

Ratings scales constitute a convenient "first step" of assessment data indicating ADHD symptomatology and combined parent and teacher rating scales based on DSMIV criteria may have a considerable predictive validity ${ }^{11,21}$. In the present study, out of 42 children who had been screened positive for the diagnosis of ADHD by parent and teachers rating scales, the diagnosis was confirmed for 31 . For the remaining 11 children ADHD manifestations raised as a consequence of other primary disorders. Our study thus confirmed that positive screening results, even if not ultimately confirmed as ADHD, do suggest conditions leading to ADHD symptomatology.

The present study indicated that ADHD combined type is the most common form of ADHD, followed by ADHD-predominantly hyperactive - impulsive and inattentive subtype. These results come in accordance with other epidemiological studies indicating ADHD-combined type as the most prevalent among children 8-10 years old ${ }^{22}$.

In conclusion, our preliminary report supports the utility of ADHD screening test in school children. Positive questionnaires by both teachers and parents should be interpreted as suggesting ADHD or another primary disorder and as indicating children who need a full clinical evaluation. Our findings still confirm that clinical evaluation by a specialized physician remains the "gold standard" for the diagnosis of ADHD.

Due to lack of a widely accepted form for conducting clinical examination of the child, the majority of studies in ADHD literature don't use this practice in their diagnostic approach. Undoubtedly, future studies must focus on this important part of ADHD diagnosis.

\section{Limitations}

A limitation of the present study is that the sample was very small, as several children initially screened positive were not available for the confirmatory clinical evaluation. Additionally, children screened negative were not called for clinical evaluation, hence sensitivity, specificity and negative predictive value of the screening procedure cannot be evaluated and no ROC curve is possible here; positive predictive value estimated at $73.8 \%$, which is relatively good.

A further limitation of the study is that the person who conducted the clinical evaluation was not blind to the fact that children being evaluated had all been screened positive for ADHD.

\section{References}

1. Baumgaertel A, Wolraich ML, Dietrich M. Comparison of diagnostic criteria for attention deficit disorders in a German elementary school sample. J Am Acad Child Adolesc Psychiatry 1995; 34(5): 629-638.

2. Skounti M, Philalithis A, Galanakis E. Variations in prevalence of attention deficit hyperactivity disorder worldwide. Eur J Pediatr 2006; 166(2): 117-123.

3. Scahill L, Schwab-Stone M. Epidemiology of ADHD in school-age children. Child Adolesc Psychiatr Clin N Am 2000; 9(3): 541-555.

4. American Psychiatric Association. The Diagnostic and Statistical Manual of Psychiatric Disorders, $4^{\text {th }}$ edition (DSM-IV). Washington, DC: APA Publishing; 1994.

5. Anastopoulos AD, Shelton TL. Assessing attention deficit/ hyperactivity disorder. New York: Kluwer Academic/Plenum Press; 2001.

6. Biederman J, Faraone SV. Attention-deficit hyperactivity disorder. Lancet 2005; 366(9481): 237-248.

7. Barkley RA. Issues in the diagnosis of attentiondeficit/hyperactivity disorder in children. Brain Dev 2003; 25(2): 77-83. 
8. Greenhill LL. Diagnosing attention-deficit/hyperactivity disorder in children. J Clin Psychiatry 1998; 59 (Suppl 7): S31-S41.

9. Wolraich ML, Baumgaertel A. The practical aspects of diagnosing and managing children with attention deficit hyperactivity disorder. Clin Pediatr (Phila) 1997; 36(9): 497-504.

10. Collett BR, Ohan JL, Myers KM. Ten-year review of rating scales. V: scales assessing attention-deficit/hyperactivity disorder. J Am Acad Child Adolesc Psychiatry 2003; 42(10): 1015-1037.

11. Hinshaw S. Attention deficits and hyperactivity in children. New York: Sage publications; 1994.

12. Rowland AS, Lesesne CA, Abramovitz AJ. The epidemiology of attention deficit hyperactivity disorder (ADHD): A public health view. Ment Retard Dev Disabil Res Rev 2002; 8(3): 162-170.

13. Skounti M, Philalithis A, Mpitzaraki K, Vamvoukas M, Galanakis E. Attention deficit hyperactivity disorder in schoolchildren in Crete. Acta Paed 2006; 95(6): 658-663.

14. Gilliam JE. Attention deficit hyperactivity disorder test. A method for identifying individuals with ADHD. Examiner's manual. Austin, Texas: PRO-ED; 1995.

15. Lerner J. Learning disabilities. Boston: Houghton Mifflin Company; 2003.

16. Lerner J. Learning disabilities: Theories, diagnosis and treatment strategies. Boston: Houghton Mifflin Company; 1993.

17. Barkley A. Attention deficit hyperactivity disorder: A handbook for diagnosis and treatment. USA: The Guilford Press; 1998.
18. Achenbach TM, Rescorla LA. Manual for school age questionnaires and profiles of Achenbachs' empirical system evaluation. Athens: Ellinika Grammata; 2003 (in Greek)

19. Power TJ, Doherty BJ, Panichelli-Mindel SM, Karustis JL, Eiraldi RA, Anastopoulos AD, et al. The predictive validity of parent and teacher reports of ADHD symptoms. J Psychopathol Behav Assess 1998; 20: 57-80.

20. American Psychiatric Association. AACAP Official Action. Summary of the practice parameters for the assessment and treatment of children, adolescents, and adults with ADHD. J Am Acad Child Adolesc Psychiatry 1997; 36(9): 1311-1317.

21. Biederman J, Keenan K, Faraone SV. Parent-based diagnosis of attention deficit disorder predicts a diagnosis based on teacher report. J Am Acad Child Adolesc Psychiatry 1990; 29(5): 698-701.

22. Anastopoulos AD, Shaffer S. Attention deficit/hyperactivity disorder. In: Walker CE, Roberts MC, eds. Handbook of clinical child psychology. New York: John Wiley\&Sons; 2000. p. 470-494.

Address for correspondence:

Dr Maria Skounti

$2^{\text {nd }}$ Special Primary School

71305 Heraklion Crete

Greece

Tel: +30 $6932214222 \&$ +30 2810326828

Fax: $+302810223424 \& 2810394606$

Email: skountim@med.uoc.gr 